\title{
Monolayers Langmuir-Blodgett Films of Synthetic Artificial Mimic Molecules That Resemble the Following Tetraether Lipids on Silicon Wafers
}

\author{
Sri Vidawati ${ }^{1}$, Ulrich Rothe ${ }^{2}$ \\ ${ }^{1}$ Department of Physics, Faculty of Natural Science and Mathematics, University Indonesia, Depok, Indonesia \\ ${ }^{2}$ Institute of Physiological Chemistry, Faculty of Medicine, University Halle-Wittenberg, Hollystr, \\ Halle/Saale, Germany \\ Email: sri.vidawati@ui.ac.id
}

Received 30 April 2015; accepted 12 June 2015; published 17 June 2015

Copyright (C) 2015 by authors and Scientific Research Publishing Inc.

This work is licensed under the Creative Commons Attribution International License (CC BY). http://creativecommons.org/licenses/by/4.0/

(c) (7) Open Access

\begin{abstract}
This study investigated the behavior and molecular organization of synthetic artificial mimic molecules that resemble the following tetraether lipids: di-0-hexadecyl-glycero-3-phosphatidyl-glycerol (DHGPG) and bis-4-dodecylphenyl-12-phosphate. These molecules were analyzed using Langmuir film balance, ellipsometry and atomic force microscopy. The monolayer LangmuirBlodgett films of DHGPG and bis-4-dodecylphenyl-12-phosphate were stable on the solid surface silicon wafers. The ellipsometry and AFM results showed that monolayers Langmuir-Blodgett films of DHGPG and bis-4-dodecylphenyl-12-phosphate were present, and the thickness of the observed films varied from $1.2-5.0 \mathrm{~nm}$.
\end{abstract}

\section{Keywords}

Di-0-Hexadecyl-Glycero-3-Phosphatidyl-Glycerol (DHGPG), Bis-4-Dodecylphenyl-12-Phosphate, Langmuir-Blodgett Film, Ellipsometry, Atomic Force Microscopy (AFM)

\section{Introduction}

The molecular organization of Langmuir-Blodgett films on the solid surface has applications in a variety of fields in nanotechnology [1], materials science [2] and biochemistry [3]. Monolayer/bilayer of bolaamphiphiles have attracted people's interest for several years because these films provide a key model for understanding the 
molecular orientation and packing mode of bipolar molecules at an air-water interface. These natural bolaamphiphiles and resembling synthetic analogues can made lipid membranes that are stable under some conditions. The extremely stable biomolecules manufactured by organisms from extreme environments have significant scientific and engineering potential for applications as novel lubricants and monolayer/bilayer lipid sensor devices.

The preparation of arranged films using Langmuir-Blodgett technology is established on the organic molecules, such as lipids or phospholipids, to form a monolayer at the air-water interfaces. The stability and homogeneity of monolayer film is a precondition for the formation of organized lipid Langmuir-Blodgett films with excellent structural integrity. Langmuir-Blodgett film methods have significant advantages in the study of the air-water and as air-solid interfacial behavior of thin solid films. Irwing Langmuir established the first systematic studies of floating surfactant monolayer. However, the first detailed description of sequential monolayer transfer was developed several years later by Katherine Blodgett. These built-up thin films are therefore known as Langmuir-Blodgett films. The most important characteristic of the monolayer properties of bolaamphiphiles is performed by measuring the surface pressure as a function of the area of the water surface, which is performed at constant temperature and is known as the surface pressure-area isotherm.

Compressed and expanded films formed from bolaamphiphiles at an air-water interface have been previously investigated by Langmuir-Blodgett film equipment. The dimensions of lipids are sufficient to form amonolayer/ bilayer with a thickness similar to that of common bilayer membranes [4]. Synthetic single-chain bolaamphiphiles are relatively flexible and thus preferentially assume a horseshoe configuration at an air-water interface, at which both hydrophilic head groups are hydrated in the water phase [5]. These configurations (horseshoe and upright standing configurations) were confirmed by computerized calculations by Gabriel et al.

This paper presents, we investigated the behavior and properties of monolayer Langmuir-Blodgett films of the synthetic artificial mimic molecules that resemble the tetraether lipids di-O-hexadecyl-glycero-3-phospha-tidylglycerol and bis-4-dodecylphenyl-12-phosphate using AFM and ellipsometry. Di-O-hexadecyl-glycero-3-phosphatidyl-glycerol is a molecule containing small-length tetraether lipids; the other side of this molecule contains half tetraether lipids because the molecule consists of only diether lipids. Bis-4-dodecylphenyl-12-phosphate is a molecule of the same length and stiffness, with both ends of a polar head group acting as a natural tetraether lipid; the other side of this molecule contains only half of the tetraether lipid molecule because these molecules contain only a single-chain hydrocarbon. The chemical structures of di-O-hexadecyl-glycero-3-phosphatidylglycerol and bis-4-dodecylphenyl-12-phosphate are shown in Figure 1.

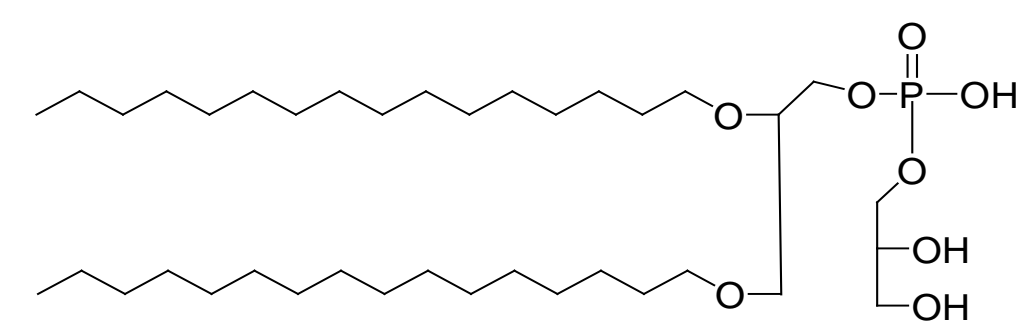

\section{di-O-hexadecyl-glycero-3-phosphatidyl-glycerol (DHGPG)}<smiles>O=P(O)(O)OCCCCCCCCCCCc1ccc(-c2ccc(CCCCCCCCCCCCP(O)(O)(O)O)cc2)cc1</smiles>

\section{bis-4-dodecylphenyl-12-phosphate}

Figure 1. Chemical structure of the synthetic artificial mimic molecules that resemble the following tetraether lipids: di-O-hexadecyl-glycero-3-phosphatidyl-glycerol (DHGPG) and bis-4dodecylphenyl-12-phosphate. 


\section{Materials and Methods}

\subsection{Materials}

For all of these experiments, lipids from the archaea Sulfolobus acidocaldarius were used. The lipids were prepared from dried bacteria in accordance with the method described by Du Plesis, et al. (2008) [6]. The Langmuir-Blodgett film experiments were performed on di-O-hexadecylglycero-3-phosphatidyl-glycerol and bis-4dodecylphenyl-12-phosphate of the highest purity. All other chemicals were purchased from Merck (Damstadt) or Sigma (Deisenhofen).

\subsection{Langmuir-Blodgett Film Methods}

The Langmuir film investigations were conducted using a commercial film balance (R \& K GmbH, Mainz, Germany) equipped with a rectangular thermostated Teflon trough (area of $418 \mathrm{~cm}^{2}$, depth of $1 \mathrm{~cm}$ ). The surface pressure was measured using a filter paper Wilhelmy balance. For the film balance experiments, the lipids were dissolved in 2:1 (v: v) chloroform/methanol at a concentration of $1.0 \mathrm{mmol} / \mathrm{L}$. The subphase was Milli Q pure water (18.2 M $2, \mathrm{pH}$ 5.6). All of the experiments were carried out at a temperature of $19^{\circ} \mathrm{C} \pm 1^{\circ} \mathrm{C}$. The lipid solution in chloroform was spread onto the subphase.

A $50 \mu \mathrm{l}$ of $1 \mathrm{~m}$ MDHGPG and $70 \mu \mathrm{l}$ of $1 \mathrm{mM}$ bis-4-dodecylphenyl-12-phosphate were used for each isotherm experiment. After spreading, the lipid films were equilibrated without surface pressure before starting the measurements. All of the films were compressed with a constant velocity of $0.045 \mathrm{~cm}^{2} \cdot \mathrm{s}^{-1}\left(2.5 \times 10^{-2} \mathrm{~nm}^{2} \cdot \mathrm{s}^{-1}\right.$ per molecule). Y-type transfer (on the upward stroke) can be used on either hydrophilic or aminosilanized substrates with a reversed head-tail arrangement, whereas X-type transfer (on the downward stroke) is better on hydrophobic substrates.

\subsection{Silicon Wafer}

Silicon wafers were used as the substrate for all of the experiments. Silicon wafers were cut to dimensions of 1 $\mathrm{cm} \times 1 \mathrm{~cm}$. We used the Piranha method to clean the silicon wafer substrates. Piranha solution is a mixture of concentrated sulfuric acid and hydrogen peroxide. The silicon wafers were cleaned by immersion in 7:3 concentrated $\mathrm{H}_{2} \mathrm{SO}_{4}: \mathrm{H}_{2} \mathrm{O}_{2}$ for 20 min and then rinsed with pure water. Thereafter, the silicon wafers were dried in a $\mathrm{N}_{2}$ gas flow.

Hydrophilic surfaces on the silicon wafers were prepared using a UV lamp (Xeradex, Lampenwerk Wipperfürth, Germany) for 5 minutes to activate the silicon wafer surface. From this method, the water contact angle changes from $48^{\circ}$ to $<20^{\circ}$ (unmodified silicon wafers).

Hydrophobic surfaces on the silicon wafers were achieved by hydrophobic silanization. The silicon wafer surfaces were activated using hydrophobization with $20 \mu$ of dimethyl C18-chlorosilane, which was placed on the siliconwafer, and the siliconwafer was then covered with a second siliconwafer that was flipped upside down. These siliconwafers were maintained at $70^{\circ} \mathrm{C}$ overnight and then washed with chloroform in an ultrasonic bath. By this method, the layer thickness was approximately $1.5 \mathrm{~nm}$, and the contact angle changed to approximately $90^{\circ}$.

Amino-silanized surfaces on the silicon wafers were prepared using a UV lamp (Xeradex, Lampenwerk Wipperfürth, Germany) for 5 minutes, and the silicon wafers were then submerged in $1 \%$ aminopropyldimethylethoxysilane in chloroform at $70^{\circ} \mathrm{C}$ overnight and washed with chloroform in an ultrasonic bath several times. The amino-silanized substrates had a layer thickness of approximately $1 \mathrm{~nm}$, and the contact angle changed to approximately $70^{\circ}$.

\subsection{Ellipsometry Measurements}

The thicknesses of all of the monolayer Langmuir-Blodgett films were characterized by ellipsometry. The lipids were analyzed after transfer on the various modified silicon wafer. The ellipsometry measurements were performed with a mapping single wavelength ellipsometer SE-400 (Sentech Instruments, Berlin, Germany). Such the light source, a He/Ne laser with a wavelength of $\mathrm{l}=623.8 \mathrm{~nm}$ was used. The angle of incidence was $60^{\circ}$. For the measurements, silicon wafers $(1 \mathrm{~cm} \times 1 \mathrm{~cm})$ with a natural oxide layer (thickness $2.6-2.9 \mathrm{~nm}$ ) and optical parameters of $\mathrm{ns}=3.828$ and $\mathrm{ks}=-\mathrm{i} 0.200$ were used. The refractive index of the tetraether lipids was established to be nu $=1.58$. As a special scanning technique, the line scan method was used for the present measure- 
ments. The surface was scanned in a single line from the pure silicon oxide layer to the lipid layer. The distance between the measurement points was $200 \mu \mathrm{m}$, and the measured area of one detected point was $250 \mathrm{\mu m}^{2}$. For the correct determination of the mean film thickness, an area of $1 \mathrm{~mm}^{2}$ in the middle of the lipid-covered silicon wafer was scanned by mapping ellipsometry. The ellipsometry data yielded the mean film thickness over a largespot area of $250 \mu^{2}$ per scanned point. The presented values were computed according to the statistical standard deviation method.

\subsection{Atomic Force Microscopy Measurements}

The surface morphology of the monolayer film was analyzed using Atomic force microscopy. Atomic force microscopy was accomplished using a NanoWizard apparatus (JPK instruments, Berlin, Germany) under atmospheric conditions. Commercial pyramidal $\mathrm{Si}_{3} \mathrm{~N}_{4}$ tips (NSC16AlBS, Micromasch, Estonia) mounted on a cantilever with a length of $230 \mu \mathrm{m}$, a resonance frequency of approximately $170 \mathrm{kHz}$ and a nominal force constant of approximately $40 \mathrm{~N} / \mathrm{m}$ were used.

The measurements were accomplished intermittently to protect sample damage. The scan size and scan frequency were using from 0.5 to $1.5 \mathrm{~Hz}$. AFM image characterization was accomplished by showing the signal height in the retrace direction $(512 \times 512$ pixels $)$.

\section{Results}

\subsection{Langmuir Films}

The phenomena of the compression-decompression cycles of a Langmuir film at an air-water interface provide detailed information on film stability, the thermodynamics and the kinetics of film formation [7]. The pressure vs. area/molecule (л/A) isotherms for the compression-decompression cycles of the monolayer Langmuir films of the synthetic artificial mimic molecules that resemble tetraether lipids DHGPG are shown in (Figure 2). The dynamic compression-decompression cycles of the monolayer Langmuir film of DHGPG were measured of concentrations $50 \mu \mathrm{l}$ at $1 \mathrm{mM}$.

As shown in Figure 2, the first compression-decompression cycle of the monolayer Langmuir film of DHGPG (50 $\mu \mathrm{l}$ of $1 \mathrm{mM}$ ) was compressed at a surface pressure coming the collapse pressure, and then decompressed to a zero surface pressure, obtaining a hysteresis-curve. After the first cycle, the lipid films were equilibrated without a surface pressure for $1 \mathrm{~h}$ before a second cycle was started. The hysteresis-curve of the monolayer Langmuir films (50 $\mu \mathrm{l}$ of $1 \mathrm{mM}$ DHGPG) were accomplished after the second cycles, and the resulting hysteresis-curves showed information for the thermodynamic and the stability of the monolayer DHGPG films [7]-[9].

\section{Langmuir film of DHGPG}

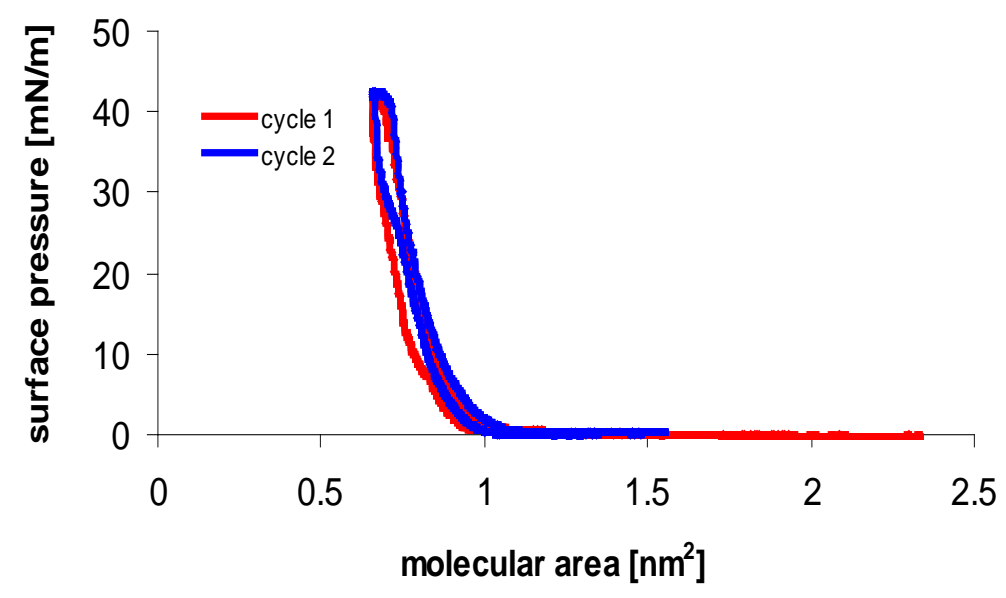

Figure 2. Representative surface pressure-area isotherms for the compression decompression cycles 1 and 2 of the monolayer Langmuir film of DHGPG at $19^{\circ} \mathrm{C}$ and $\mathrm{pH} 5.5$. 
The stability of the monolayer Langmuir film of bis-4-dodecylphenyl-12-phosphate was low (data not shown). It was not possible to compress and decompress the film. However, we attempted to transfer the monolayer Langmuir film of bis-4-dodecylphenyl-12-phosphate $(70 \mu \mathrm{l}$ of $1 \mathrm{mM})$ to a solid substrate on modified silicon wafers.

Surface isotherms are usually used to describe the strength and stability of Langmuir-Blodgett films. All of the transfer experiments (Y-type and X-type) of the Langmuir-Blodgett films were accomplished immediately before the collapse pressure was achieved. The X-type film transfers method was used for hydrophobically modified surfaces. Y-type film transfers method was used for hydrophilically modified and amino-silanized modified surfaces.

\subsection{Ellipsometry}

The thicknesses of all of the monolayer Langmuir-Blodgett films were measured by ellipsometry. Ellipsometry data gives the mean film thickness over a large spot area of $250 \mu^{2}$ and therefore cannot separate between films with different morphological structures. The Langmuir-Blodgett films were observed on various modified silicon wafer, and different thickness values were produced.

The thickness of the monolayer Langmuir-Blodgett films of DHGPG on the hydrophilic modified silicon wafer and on the hydrophobic modified silicon wafer showed thickness values were only $1.2 \mathrm{~nm}$ and $3.0 \mathrm{~nm}$, respectively [8]-[10].

The thickness of the monolayer Langmuir-Blodgett films of bis-4-dodecylphenyl-12-phosphate gave values between $3.5 \mathrm{~nm}$ for the amino-silanized modified silicon wafer and $3.5 \mathrm{~nm}$ for the hydrophobic modified silicon wafer [8]-[10].

\subsection{Atomic Force Microscopy}

AFM is used for studied and analyzed the surface morphology and thickness of the monolayer Langmuir-Blodgett films of DHGPG and bis-4-dodecylphenyl-12-phosphate on the molecular scale. Typical AFM images of monolayer DHGPG films after transfer on the hydrophobic and hydrophilic modified silicon wafers are displayed in Figure 3(a) and Figure 3(b). From the AFM images, the thicknesses of the monolayer LangmuirBlodgett films of DHGPG on the hydrophilic of modified silicon wafer (Y-type transfer) and hydrophobic of modified silicon wafer (X-type transfer) were found to be approximately 4.0 - $5.0 \mathrm{~nm}$ [8] [9].

The monolayer Langmuir-Blodgett films of bis-4-dodecylphenyl-12-phosphate on hydrophobic of modified silicon wafer (X-type transfer) and amino-silanised of modified silicon wafer (Y-type transfer) were evaluated by AFM (see Figure 4(a) and Figure 4(b)). The thicknesses of the monolayer Langmuir-Blodgett films of bis4-dodecylphenyl-12-phosphate were 4.0 - $5.0 \mathrm{~nm}$ [8] [9]. All of the monolayer Langmuir-Blodgett film information was for an upright standing configuration [5] [11].

\section{Discussion}

The study of monolayer/bilayer formation is an interesting branch of physical chemistry that has applications in many fields of science and technology. A thorough understanding of monolayer/bilayer behavior is important for utilizing the Langmuir-Blodgett method, which implicates the position of a film on a water surface with following transfer onto a solid substrate.

The surface isotherms are usually used to describe the strength and stability of Langmuir-Blodgett films. When compression-decompression cycles where measured; statements about the film stability and the kinetics of the film formation are available.

The Langmuir film stability of DHGPG at the air-water interface is shown on a hysteresis-curve from the surface pressure-area ( $\pi$-A) isotherms (Figure 2). A hysteresis loop was performed for $50 \mu \mathrm{l}$ of $1 \mathrm{mM}$ concentration of lipid solutions. The concentration of DHGPG is also an important parameter for obtaining the stability of the Langmuir films. The dynamic compression-decompression isotherm cycles of the synthetic artificial mimic molecules that resemble the following tetraether lipids: DHGPG Langmuir films provide complete information on the formation, molecular area, stability, collapse behavior, phase transitions, thermodynamics, and kinetics of film formation [7] [9].

The pressure versus area/molecule (л/A) isotherms were recorded at $19^{\circ} \mathrm{C} \pm 1^{\circ} \mathrm{C}$ and $\mathrm{pH} 5.5$ for $50 \mu 1$ of 1 
mM concentration of DHGPG; the results are shown in Figure 2. DHGPG (50 $\mu \mathrm{l}$ of $1 \mathrm{mM})$ had a collapse film pressure of approximately $41 \mathrm{mN} / \mathrm{m}$ and a molecular area from $0.60 \mathrm{~nm}^{2}$ to $0.75 \mathrm{~nm}^{2}$.

The compression and decompression cycles can be essential to reach consistent isotherms stability of the Langmuir films. Highly stable Langmuir films are necessary for finding out the quality of the Langmuir-Blodgett monolayer. Figure 2 shows the Langmuir films of $50 \mu \mathrm{l}$ of $1 \mathrm{mM}$ DHGPG. The first cycle had the highest surface pressure at approximately $41 \mathrm{mN} / \mathrm{m}$ and a molecular area from $0.60 \mathrm{~nm}^{2}$ to $0.75 \mathrm{~nm}^{2}$. The second cycle also displayed the most stable surface pressure of the Langmuir film of DHGPG of $41 \mathrm{mN} / \mathrm{m}$ and a molecular area that was almost equal to that observed in the first cycle (between $0.60 \mathrm{~nm}^{2}$ and $0.75 \mathrm{~nm}^{2}$ ). As shown in Figure 2, the curves show the thermodynamic features and the excellent stability of the Langmuir film of DHGPG at an air-water interface. The floating monolayer stability is a significant parameter for obtaining veryhigh-quality Langmuir-Blodgett films. Some efforts have been done to produce high-quality Langmuir-Blodgett films of bolaamphiphiles [8]-[10] [12]-[16].

The data characterized by ellipsometry for $50 \mu \mathrm{l}$ of $1 \mathrm{mM}$ DHGPG had a high collapse pressure of $41 \mathrm{mN} / \mathrm{m}$ and a molecular area approximately $0.60 \mathrm{~nm}^{2}$. The mean thickness of the film was $3.5 \mathrm{~nm}$ after hydrophobic transfer (X-type), which suggested an upright standing conformation, whereas a mean thickness of the film of only $1.2 \mathrm{~nm}$ was measured after hydrophilic transfer (Y-type), which suggested a horse shoe conformation. One could hypothesize that the existence of the films on the solid substrate would be expected after hydrophobic and hydrophilic transfer.

The thickness of the monolayer Langmuir-Blodgett films of $70 \mu \mathrm{l}$ of $1 \mathrm{mM}$ bis-4-dodecylphenyl-12-phosphate was measured by ellipsometry. The ellipsometry measurements after transfer Langmuir monolayer film on the hydrophobic surface (X-type transfer) yielded a film height of approximately $3.5 \mathrm{~nm}$, which suggested an upright standing conformation. The thickness of the Langmuir monolayer film transferred onto amino-silanized surface (Y-type transfer) was approximately $3.5 \mathrm{~nm}$, which also indicative an upright standing conformation

Ellipsometry, as a mapping technique, will cover a spot area of approximately $250 \mu^{2}$. The ellipsometry data for the monolayer Langmuir-Blodgett films of bis-4-dodecylphenyl-12-phosphate transferred onto hydrophobic and amino-silanized of modified silicon wafers were appropriate with an upright standing confirmation, and the monolayer Langmuir-Blodgett films of DHGPG transferred onto hydrophobic and hydrophilic of modified silicon wafers varied in thickness between $1.2 \mathrm{~nm}$ and $3.5 \mathrm{~nm}$.

The AFM results (Figure 3(a) and Figure 3(b)) for the monolayer Langmuir-Blodgett film of $50 \mu \mathrm{l}$ of $1 \mathrm{~m}$ MDHGPG revealed a relatively homogeneous lipid layer. On the hydrophilic (Y-type transfer) surface of modified silicon wafer, the film had a thickness of approximately $4.0-5.0 \mathrm{~nm}$, which suggested an upright stand- ing conformation, whereas $50 \mu \mathrm{l}$ of $1 \mathrm{mM}$ DHGPG on a hydrophobic surface (X-type transfer) looked like a collection of small flat islands with a thickness of approximately $4.0-5.0 \mathrm{~nm}$, which also suggested an upright standing conformation. These results suggest that the monolayer Langmuir-Blodgett films of $50 \mu \mathrm{l}$ of $1 \mathrm{mM}$ DHGPG on the hydrophilic and hydrophobic surfaces of modified silicon wafers were stable.

Based on the AFM characterization (Figure 4(a) and Figure 4(b)), the monolayer Langmuir-Blodgett film of $70 \mu \mathrm{l}$ of $1 \mathrm{mM}$ bis-4-dodecylphenyl-12-phosphate on the amino-silanized surface of modified silicon wafer (Y type transfer) was stable with a thickness of approximately $4.0-5.0 \mathrm{~nm}$. The AFM images of the monolayer Langmuir-Blodgett films show some islands and domains. On the hydrophobic surface of modified silicon wafer (X-type transfer), the film of $70 \mu \mathrm{l}$ of $1 \mathrm{mM}$ bis-4-dodecylphenyl-12-phosphate had a thickness of approximately 3.0 - $4.0 \mathrm{~nm}$, indicating an upright standing configuration.

\section{Conclusions}

Monolayer Langmuir-Blodgett film studies of di-O-hexadecyl-glycero-3-phosphatidyl-glycerol and bis-4-dodecylphenyl-12-phosphate on silicon wafers are presented in this manuscript. The results show that monolayer films of DHGPG and bis-4-dodecylphenyl-12-phosphate are present, and the thicknesses of the observed films vary between 1.2 and $5.0 \mathrm{~nm}$.

By summarizing our data, we show that the relatively stiff synthetic artificial mimic molecules that resemble the tetraether lipids di-O-hexadecyl-glycero-3-phosphatidyl-glycerol and bis-4-dodecylphenyl-12-phosphate can adopt several conformational supra molecular arrangements. All of the monolayer Langmuir-Blodgett films of di-O-hexadecyl-glycero-3-phosphatidyl-glycerol and bis-4-dodecylphenyl-12-phosphate are stable on a solid surface. Further study is necessary to enable the optimization of monolayer films for biomedical applications. 


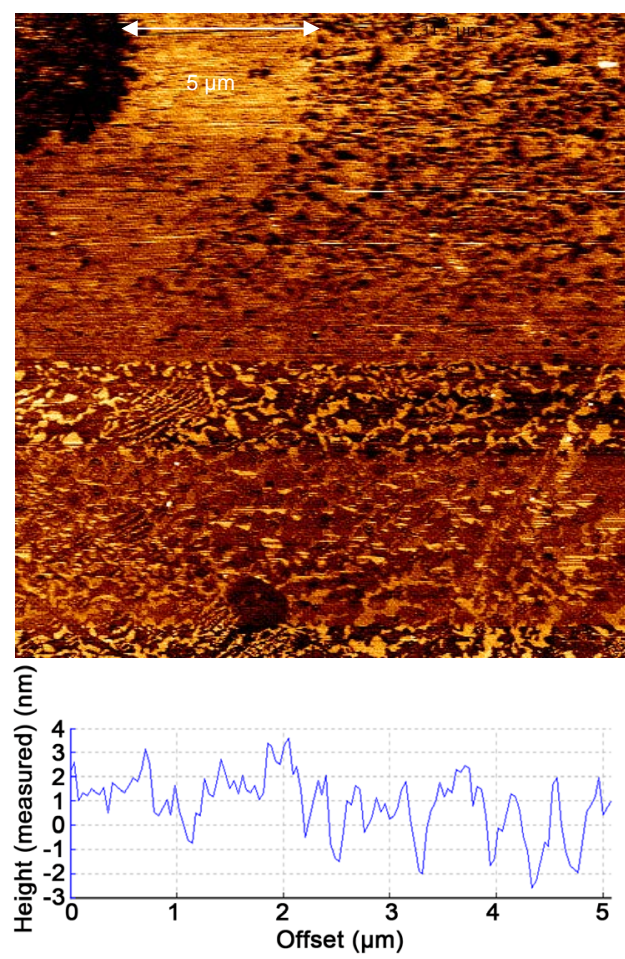

(a)
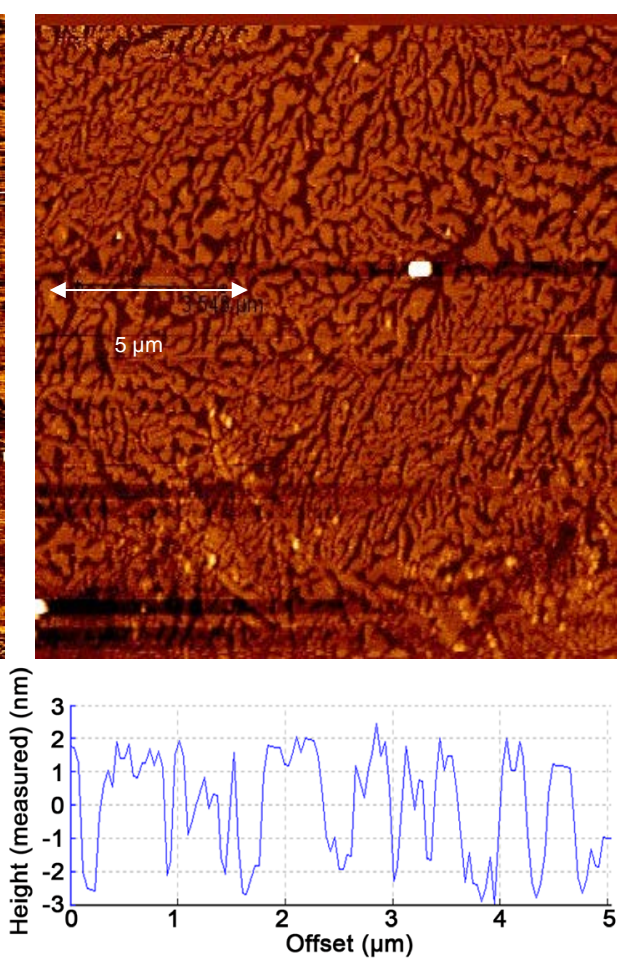

(b)

Figure 3. AFM images of the monolayer Langmuir-Blodgett films of DHGPG. (a) DHGPG hydrophilic surface (Y-type transfer); (b) DHGPG hydrophobic surface (X-type transfer).
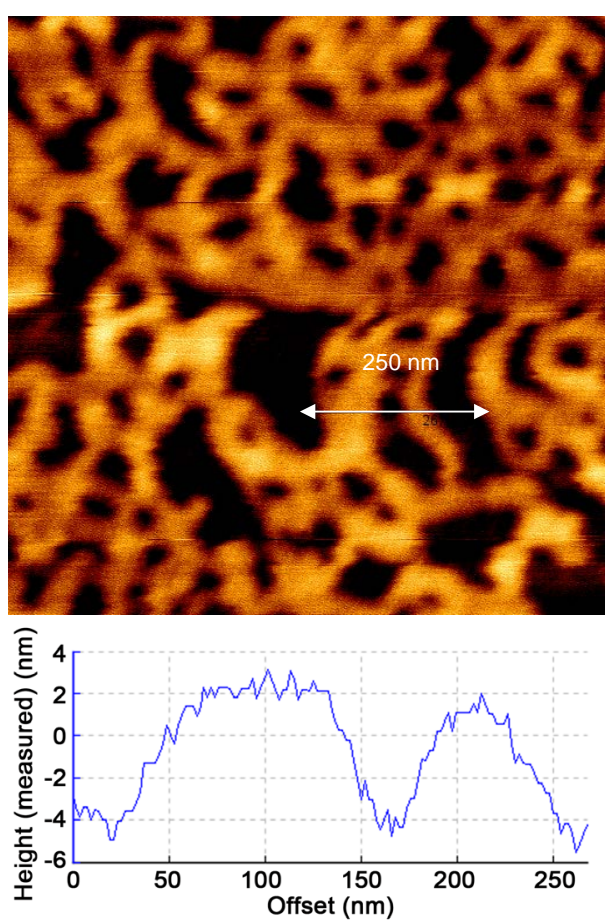

(a)
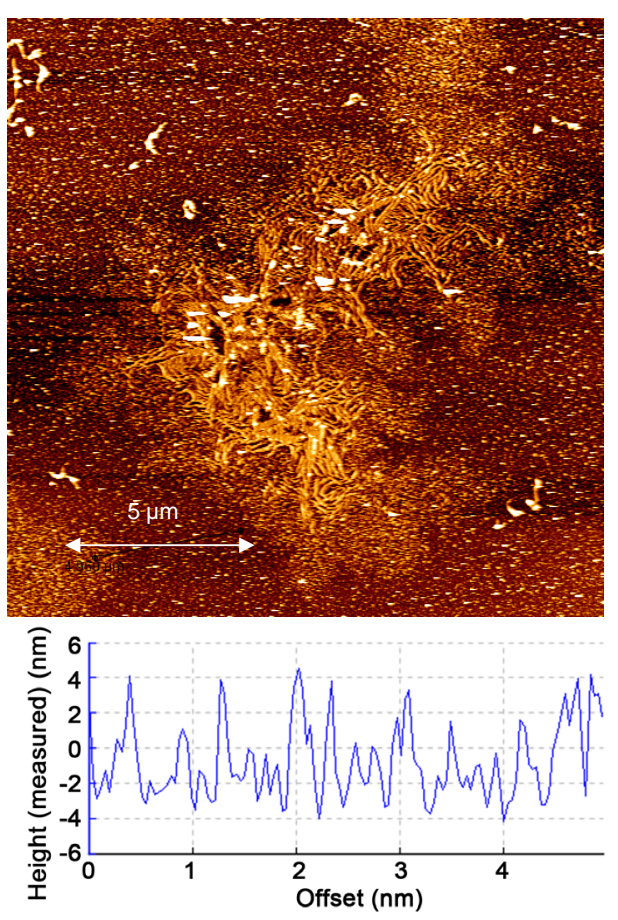

(b)

Figure 4. AFM images of the monolayer Langmuir-Blodgett films of bis-4-dodecylphenyl-12-phosphate: (a) bis-4-dodecylphenyl-12-phosphate amino-silanized surface (Y-type transfer) and (b) bis-4-dodecylphenyl-12-phosphate hydrophobic surface (X-type transfer). 


\section{Acknowledgments}

This study was supported by the Deutscher Akademischer Austauschdienst (DAAD, SV).

\section{References}

[1] Weissbuch, I., Baxter, P.N.W., Cohen, S., Cohen, H., Kjaer, K., Howes, P.B., Als-Nielsen, J., Hanan, G.S., Schubert, U.S., Lehn, J.-M., Leiserowitz, L. and Lahav, M.J. (1998) Self-Assembly at the Air-Water Interface. In-Situ Preparation of Thin Films of Metal Ion Grid Architectures. Journal of the American Chemistry Society, 120, 4850-4860. http://dx.doi.org/10.1021/ja980205w

[2] Swift, J.A., Pivovar, A.M., Reynolds, A.M. and Ward, M.D. (1998) Template-Directed Architectural Isomerism of Open Molecular Frameworks: Engineering of Crystalline Clathrates. Journal of the American Chemistry Society, 120, 5887-5894. http://dx.doi.org/10.1021/ja980793d

[3] Sengupta, K., Schilling, J., Marx, S., Fischer, M., Bacher, A. and Sackmann, E. (2003) Mimicking Tissue Surfaces by Supported Membrane Coupled Ultrathin Layer of Hyaluronic Acid. Langmuir, 19, 1775-1781. http://dx.doi.org/10.1021/la026146o

[4] Stern, J., Freisleben, H.J., Janku, S. and Ring, K. (1992) Black Lipid Membranes of Tetraether Lipids from Thermoplasma acidophilum. Biochimica et Biophysica Acta, 1128, 227-236. http://dx.doi.org/10.1016/0005-2760(92)90312-J

[5] Fuhrhop, J., David, H.H., Mathieu, J., Liman, U., Winter, H.J. and Boekema, E.J. (1986) Bolaamphiphiles and Monolayer Lipid Membranes Made from 1,6,19,24-Tetraoxa-3,21-cyclohexatriacontadiene-2,5,20,23-tetrone. Journal of the American Chemistry Society, 108, 1785-1791. http://dx.doi.org/10.1021/ja00268a013

[6] Bode, M.L., Buddoo, S.R., Minnaar, S.H. and Du Plessis, C.A. (2008) Extraction, Isolation and NMR Data of the Tetraether Lipid Calditoglycerocaldarchaeol (GDNT) from Sulfolobus metallicus Harvested from a Bioleaching Reactor. Chemistry and Physics of Lipids, 154, 94-104. http://dx.doi.org/10.1016/j.chemphyslip.2008.02.005

[7] Vidawati, S., Rothe, U. and Bakowsky, U. (2012) Langmuir Films Stability Phenomenon of Glycerol Dialkyl Nonitol Tetraether at the Air-Water Interface for Variations Spreading Time. Advances in Biological Chemistry, 2, $233-237$. http://dx.doi.org/10.4236/abc.2012.23028

[8] Vidawati, S., Sitterberg, J., Bakowsky, U. and Rothe, U. (2010) AFM and Ellipsometric Studies on LB Films of Natural Asymmetric and Symmetric Bolaamphiphilic Archaebacterial Tetraether Lipids on Silicon Wafers. Colloids and Surfaces B: Biointerfaces, 78, 303-309. http://dx.doi.org/10.1016/j.colsurfb.2010.03.015

[9] Vidawati, S., Sitterberg, J., Rothe, U. and Bakowsky, U. (2011) Stability of Monomolecular Films of Archaebacterial Tetraether Lipids on Silicon Wafers: A Comparison of Physisorbed and Chemisorbed Monolayers. Colloids and Surfaces B: Biointerfaces, 87, 209-216. http://dx.doi.org/10.1016/j.colsurfb.2011.05.005

[10] Bakowsky, U., Rothe, U., Antonopoulos, E., Martini, T., Henkel, L. and Freisleben, H.J. (2000) Monomolecular Organization of the Main Tetraether Lipid from Thermoplasma acidophilum at the Water-Air Interface. Chemistry and Physics of Lipids, 105, 31-42. http://dx.doi.org/10.1016/S0009-3084(99)00131-0

[11] Gabriel, J.L., Chong, P.L.G. (2000) Molecular Modeling of Archaebacterial Bipolar Tetraether Lipid Membranes. Chemistry and Physics of Lipids, 105, 193-200. http://dx.doi.org/10.1016/S0009-3084(00)00126-2

[12] Rolandi, R., Schindler, H., De Rosa, M. and Gambacorta, A. (1986) Monolayers of Ether Lipids from Archaebacteria, European Biophysics Journal, 14, 19-27. http://dx.doi.org/10.1007/BF00260399

[13] Dote, J.L., Barger, W.R., Behroozi, F., Chang, E.L., Lo, S.L., Montague, C.E. and Nagumo, M. (1990) Monomolecular Film Behavior of Tetraether Lipids from a Thermoacidophilic Archaebacterium at the Air/Water Interface. Langmuir, 6, 1017-1023. http://dx.doi.org/10.1021/la00095a023

[14] Elferinck, M.G.L., De Wit, J.G., Demel, R., Driessen, A.J.M. and Konings, W.N.J. (1992) Functional Reconstitution of Membrane Proteins in Monolayer Liposomes from Bipolar Lipids of Sulfolobus acidocaldarius. Journal of Biological Chemistry, 267, 1375-1381.

[15] De Rosa, M. (1996) Archaeal Lipids: Structural Features and Supramolecular Organization. Thin Solid Films, 284-285, 13-17. http://dx.doi.org/10.1016/S0040-6090(96)08832-3

[16] Dante, S., De Rosa, M., Maccioni, B., Morana, A., Nicolini, C., Rustichelli, F., Troitsky, V.I. and Yang, B. (1995) Thermal, stability of bipolar lipid Langmuir Blodgett films by X-Ray diffraction. Molecular Crystals and Liquid Crystals Science and Technology, 262, 191-207. http://dx.doi.org/10.1080/10587259508033525 